\title{
Primary cerebral metastasis in bilateral breast cancer
}

\author{
B. Ataseven, A. Staunton, W. Eiermann \\ Rotkreuzklinikum München, Frauenklinik, Taxisstr, München, Germany.
}

\section{Presentation and diagnosis}

We present the case of a 53-year-old patient complaining of a left-sided breast lump, which she had first noticed 2 years back following trauma to the breast caused by a fall. She had consulted a doctor about the lump 3 days prior to attending our department. The patient denied any further symptoms or signs.

Clinically, she presented with the following findings (Fig. 1): a red, fist-sized, non-ulcerating exophytic lump protruding from the upper quadrant of the left breast. The remaining breast tissue consisted of what felt like a tumor conglomerate. The right breast was characterized by very suspicious, irregular thickened tissue extending for several centimeters. There were pathological lymph nodes palpable in both axillae.

Ultrasound examination revealed in the left breast next to the $8-9 \mathrm{~cm}$ clinically exophytic tumor, a second suspicious area measuring $8 \mathrm{~mm}$ at in the area between the left upper and lower inner quadrants approximately at $8 \mathrm{~cm}$ distance from the nipple. On the right side, ultrasound revealed a suspicious, inhomogenous area. There were bilateral enlarged supra-clavicular lymph nodes (Fig. 2), which were clinically metastatic. The patient refused mammography.

Stance biopsy revealed a right-sided invasive receptor-positive lobular carcinoma (her2 negative) and a left-sided invasive receptor negative ductal carcinoma (her2 negative).

Correspondence to: Beyhan Ataseven, Rotkreuzklinikum München, Frauenklinik, Taxisstr 3 80637, München, Germany. E-mail: beyhan. ataseven@swmbrk.de

Received: 29/05/08 Accepted: 05/06/08 $\mathrm{BCO} / 783 / 2008 / \mathrm{CS}$

\section{Treatment and outcome}

The patient requested a primary bilateral mastectomy. During the operation, multiple hard, enlarged lymph nodes were noted in both breasts.

The final histological classification was a multicentered right-sided carcinoma $(4 \mathrm{~cm})(\mathrm{pT} 2(\mathrm{~m}) \mathrm{pN} 0$ $(0 / 15)$ L0, V0, G2, R0). On the left side was an $8 \mathrm{~cm}$ carcinoma (pT4b, pN0 (0/14), L0, V0, G2, R0). The excision was histologically complete. The regional lymph nodes (15 on the right, 14 on the left) were negative on histological examination. As they were clinically highly suspicious, the tissue was sent for a second opinion at a histology reference center, where immune histochemistry was used to confirm the nodal negativity for any form of invasion. Follicular lymphatic hyperplasia of non-specific origin (Fig. 3) was reported. To exclude distant metastases, a chest X-ray, CT neck and thoracic inlet,

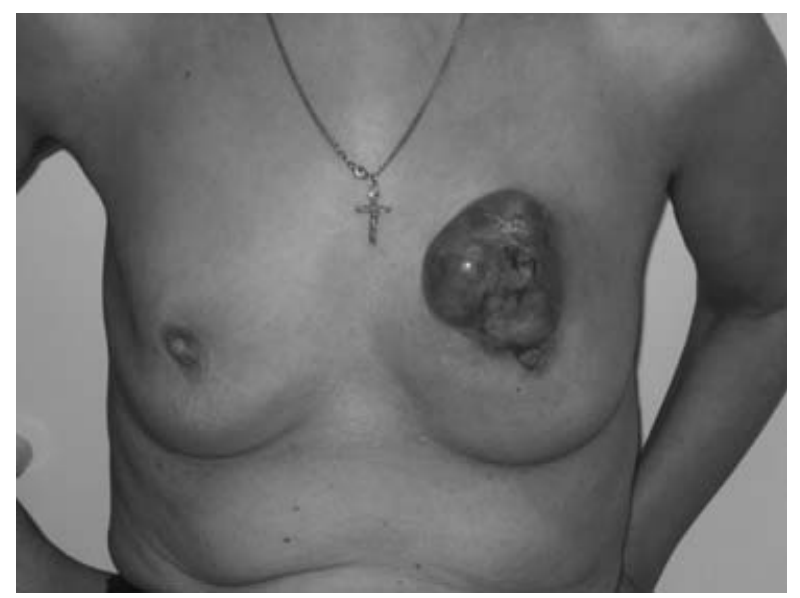

Figure 1.

Lump protruding from the upper quadrant of the left breast. 


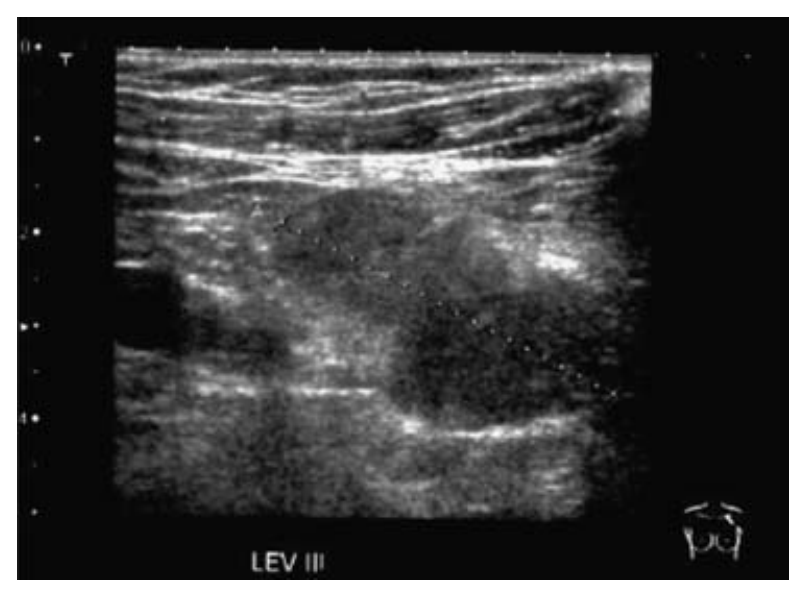

Figure 2.

Ultrasound image of pathological lymph nodes in level 3 left.

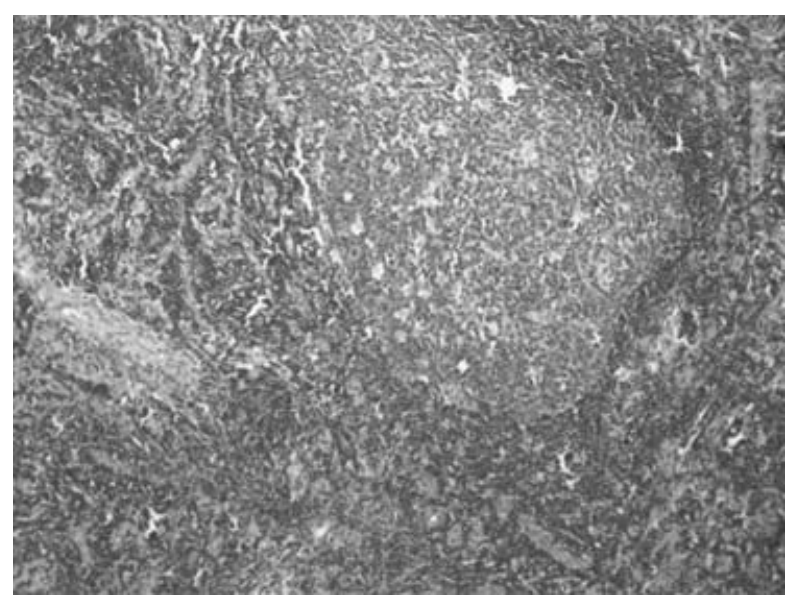

Figure 3.

Microscopic image of lymph nodes without any sign of metastasis.

skeletal scintography and a CT abdomen were performed, with no evidence of distant metastasis.

The mastectomy wounds first healed normally, but after an interval a secondary infection developed. Pseudomonas aeruginosa was isolated from the wound, which was treated by oral ciprofloxacin.

The patient underwent a generalized tonic-clonic seizure whilst attending hospital. The MRT examination showed a left-sided intracranial parietooccipital metastasis (Fig. 4). The patient's condition was stabilized and she elected to have the solitary lesion neurosurgically resected. The histology revealed a metastasis of the known breast cancer.

The results were discussed with the patient and she was offered the choice of palliative hormonal therapy with/without chemotherapy or radiotherapy. The patient refused further therapy at the time. Four

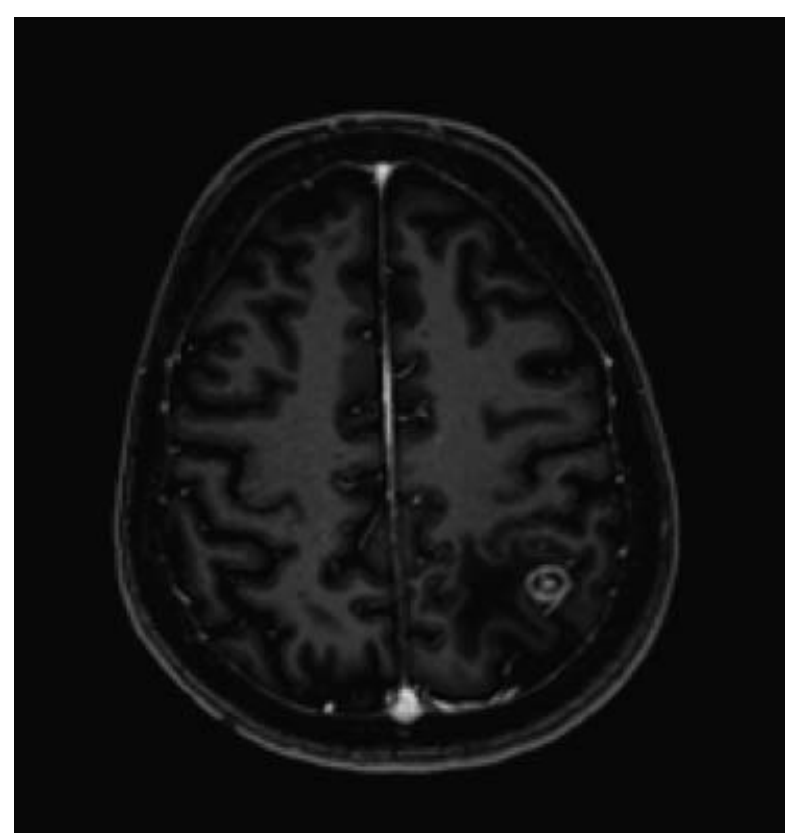

Figure 4.

Cerebral metastasis and surrounding edema.

months after the neurosurgery, further intra-cerebral metastases were found. A PET scan was performed. The patient chose surgery as a therapy option, and the histology was that of a poorly differentiated carcinoma, probably metastatic breast cancer.

\section{Comments}

The case is interesting, as it is rare to find intracerebral metastasis of breast cancer without locoregional metastatic disease occurring first. In the literature, the usual sites for metastasis are in the lung, liver or the skeletal system. We have been unable to find details of incidence of the above constellation, with intra-cerebral metastasis occurring before loco-regional metastasis. This is probably a very rare occurrence. We know that prognostic markers such as lymph node status, tumor stadium grading, hormone receptor status and Her2new status can define the risk of distant metastasis. The single most important predictive parameter (lymph node status) was negative in this case.

In summary, we now have to ask whether the standard staging procedure, which does not, in the absence of clinical signs, normally include cerebral diagnosis, should be revised in the light of the above case. In our opinion, a risk-adapted procedure should be adopted: intracranial investigation should only be entertained should the patient have signs or symptoms of cerebral pathology. 